\title{
Modelling and simulation of electric drive vehicle based on space vector modulation technique and field oriented control strategy
}

\begin{abstract}
Recently, the electric vehicle has emerged as a powerful platform for mitigating energy crisis and reducing environmental pollution in the transportation sector. The major drawbacks of electrically powered automobile that limits its competitiveness with the internal combustion engine counterpart are the vehicle driving range and battery energy capacity. Hence, limited energy storage warrants the need for an effective and efficient energy utilisation in the overall system. This paper uses Field Oriented Control algorithm and Space Vector Modulation technique to enhance and to optimise energy saving at the same time improve the vehicle induction motor efficiency. A simple electric vehicle drive with vehicle dynamic and tractive loads for motor driving load were modelled and simulated using Matlab/Simulink. Simulation results show that modelled vehicle speed satisfied the acceleration index for the electric vehicle.
\end{abstract}

Keyword: Electric vehicle; FOC; IM; PWM; SVM 